\title{
3-month surgical outcomes of Implantable Collamer Lens implantation for myopic regression after laser vision correction surgeries: a retrospective case series
}

Byunghoon Chung ${ }^{1+}$, Joon Hyun Kim ${ }^{2+}$, David S. Y. Kang ${ }^{1}$, Dong Jun Kang ${ }^{2}$, Eung Kweon Kim³, Kyoung Yul Seo ${ }^{3}$, Ikhyun Jun ${ }^{3}$ and Tae-im $\mathrm{Kim}^{3 *}$

\begin{abstract}
Background: To investigate the surgical outcomes of implantable collamer lens (ICL) implantation in eyes with residual myopia after primary laser vision correction (LVC) surgeries.

Methods: This study included patients who underwent ICL implantation and had a history of LVC surgery, including photorefractive keratectomy (PRK) or laser-assisted in situ keratomileusis (LASIK). Visual acuity and refractive error were assessed pre and 3-months postoperatively and the efficacy and safety indices calculated accordingly.

Results: A total of 30 eyes of 17 patients were included in this study. At 3 months, the mean logMAR uncorrected distance visual acuity (UDVA), corrected distance visual acuity (CDVA), and spherical equivalent were $-0.03 \pm 0.11$ (include logMAR), $-0.04 \pm 0.09$ (include logMAR), and $-0.06 \pm 0.33$ diopters (D), respectively. The 3-month Snellen UDVA was better than $20 / 20$ for $83 \%$ of eyes, and $97 \%$ of eyes showed an unchanged or improved CDVA after surgery. The mean efficacy and safety indices were $1.11 \pm 0.22$ and $1.13 \pm 0.20$, respectively. Further, 93 and 100\% of eyes were within \pm 0.5 and $\pm 1.0 \mathrm{D}$ of the attempted spherical equivalent refraction, respectively.
\end{abstract}

Conclusions: ICL implantation in eyes with myopic regression after previous LVC surgery showed safe, effective, and predictable outcomes.

Trial registration: retrospectively registered.

Keywords: Implantable collamer lens, Myopic regression, Laser-assisted in situ keratomileusis, Photorefractive keratectomy

\section{Background}

Regression following corneal refractive procedures refers to the tendency of the cornea to shift towards the preoperative refractive status after achieving desired refraction

\footnotetext{
*Correspondence: tikim@yuhs.ac

†Byunghoon Chung and Joon Hyun Kim contributed equally to this work.

${ }^{3}$ Department of Ophthalmology, Institute of Vision Research, Yonsei

University College of Medicine, 50-1, Yonsei-ro, Seodaemun-gu, Seoul 03722, Republic of Korea

Full list of author information is available at the end of the article
}

for a period of time [1]. Residual refractive errors after primary laser vision correction (LVC) surgery is the most common cause of postoperative dissatisfaction, and surgical retreatment is considered in eyes with regression. The rate of enhancement, the surgical retreatment, has been reported to be $1.8-22 \%$ for laser-assisted in situ keratomileusis (LASIK) [2-4] and 2.3-5.2\% for photorefractive keratectomy (PRK) $[5,6]$.

Enhancement options for LASIK include flap relift with ablation or recutting a new flap and surface original author(s) and the source, provide a link to the Creative Commons licence, and indicate if changes were made. The images or other third party material in this article are included in the article's Creative Commons licence, unless indicated otherwise in a credit line to the material. If material is not included in the article's Creative Commons licence and your intended use is not permitted by statutory regulation or exceeds the permitted use, you will need to obtain permission directly from the copyright holder. To view a copy of this licence, visit http://creativecommons.org/licenses/by/4.0/. The Creative Commons Public Domain Dedication waiver (http://creativeco mmons.org/publicdomain/zero/1.0/) applies to the data made available in this article, unless otherwise stated in a credit line to the data. 
ablation, and for PRK include LASIK or a new PRK procedure. However, these enhancement options are only available when the residual stromal bed is thick enough to maintain corneal biomechanical stability after the enhancement procedure. In addition, enhancement options involving corneal laser procedures pose a risk of postoperative complications, including epithelial ingrowth, subepithelial haze formation, and corneal ectasia.

Posterior chamber phakic intraocular lens implantation has been reported to be safe and effective for the correction of myopia and myopic astigmatism throughout a long-term follow-up period [7]. The implantable collamer lens (ICL) is widely used to correct moderate and high myopia, and some studies have reported ICL implantation in eyes that underwent previous corneal surgeries, including LASIK, PRK, and radial keratotomy [8-10]. Among these studies, one reported ICL implantation to be a safe and effective way to retreat residual myopia after primary corneal refractive surgeries [8]. In the present study, we aimed to analyze the surgical outcomes of ICL implantation in eyes with myopic regression after primary LVC surgeries including LASIK and PRK.

\section{Methods}

\section{Study design}

This retrospective, observational case series was conducted at Yonsei University College of Medicine, Seoul, Republic of Korea. The tenets of the Declaration of Helsinki and good clinical practice were followed, and institutional review board approval from Yonsei University College of Medicine was obtained (No. 4-2020-0396). Owing to the retrospective nature of the study, the requirement for informed consent was waived. All surgeries were performed by a single surgeon (JHK) between June 2020 and August 2020.

\section{Study population}

A total of 17 patients with residual myopia or myopic astigmatism after a previous history of LVC were enrolled. All patients decided to have ICL implantation after detailed explanation of possible enhancement options before the surgery. Other inclusion criteria were a stable refractive status for at least 1 year, a preoperative corrected distance visual acuity (CDVA) of 20/30 or better, and no sign of corneal ectasia on at least three consecutive corneal tomographic evaluations. Patients with any kind of ocular surface diseases, ocular trauma, glaucoma, cataract, an endothelial cell density $<2000$ cells/ $\mathrm{mm}^{2}$, or an anterior chamber depth from the endothelium $<2.8 \mathrm{~mm}$ were excluded.

\section{Assessment}

To evaluate outcomes, all patients were assessed before and 3 months after ICL implantation. Patients' assessments included uncorrected distance visual acuity (UCVA) and CDVA measured in logMAR, slit-lamp examination (Haag-Streit AG), keratometry, pachymetry (ARK-530A Auto Ref/Keratometer, Nidek Co., Ltd.), specular microscopy (SP-3000P, Topcon Corporation), Scheimpflug-based corneal tomography (Pentacam HR, OCULUS Optikgeräte $\mathrm{GmbH}$ ), and anterior segment optical coherence tomography (Visante, Carl Zeiss Meditec AG). The efficacy index (the ratio of postoperative UDVA to preoperative CDVA) and safety index (the ratio of postoperative CDVA to preoperative CDVA) were also estimated.

\section{Surgical procedure}

All procedures were performed through a superior $3.0-\mathrm{mm}$ corneal incision after instillation of $0.5 \%$ phenylephrine and $0.5 \%$ tropicamide (Mydrin-P, Santen Pharmaceutical Co., Ltd.) under topical anesthesia with $0.5 \%$ proparacaine (Alcaine, Alcon). Further, $1 \%$ sodium hyaluronate (Healon, Johnson \& Johnson Vision) was injected into the anterior chamber, and the ICL was inserted through an injector cartridge. After positioning the ICL, the sodium hyaluronate was completely removed by manual irrigation and aspiration. The V4c ICL model (Staar Surgical) was used in all cases. ICL powers were calculated using a modified vertex formula provided by the manufacturer. Patients were instructed to use $0.5 \%$ moxifloxacin (Vigamox, Alcon) and 1\% prednisolone (Pred Forte, Allergan, Inc.) four times a day for a week.

\section{Statistical analysis}

Data are presented as mean \pm standard deviation. IBM SPSS Statistics for Windows (v. 25.0, IBM Corporation) was used to perform statistical analyses. Data normality was confirmed using a Kolmogorov-Smirnov test. Preand postoperative measurements were compared using a paired t-test for normally distributed data and the Wilcoxon signed-rank test for non-normally distributed data. Comparisons between the two groups were performed using an independent sample t-test for normally distributed data and a Mann-Whitney U test for non-normally distributed data. The refractive predictability was analyzed using linear regression, and comparison of categorical variables was performed using a chi-squared test. A $P$-value $<.05$ was considered statistically significant. 


\section{Results}

\section{Patient characteristics}

A total of 30 eyes of 17 patients were enrolled in the present study. Patient characteristics are presented in Table 1 . The mean central corneal thickness, mean keratometry reading, and endothelial cell count were not significantly changed at 3 months after ICL implantation compared to those before the implantation. There were no intraoperative and postoperative complications, including cataract formation and elevation of intraocular pressure, in any of the cases.

\section{Visual outcomes, efficacy, and safety}

The mean UDVA and CDVA improved significantly at 3 months after ICL implantation (Table 1); $83 \%$ of eyes showed a 3-month UDVA of 20/20 or better (Fig. 1A). One eye showed loss of one Snellen line of CDVA at 3 months after ICL implantation, while the remaining eyes showed no change or gain of Snellen lines of CDVA (Fig. 1B). The mean efficacy index (ratio of postoperative UDVA to preoperative CDVA) and safety index (ratio of postoperative CDVA to preoperative CDVA) were $1.11 \pm 0.22$ and $1.13 \pm 0.20$, respectively. Additional analysis was performed according to the types of primary LVCs (PRK and LASIK; Table 2). There was no significant difference between the PRK and LASIK groups regarding UDVA, CDVA, and refractive errors.

\section{Refraction and predictability}

Refractive errors, including sphere and spherical equivalent (SEQ), decreased significantly at the 3-month postoperative evaluation, while cylindrical errors showed no significant change (Table 1). The attempted versus achieved SEQ graph showed a slope and correlation coefficient $\left(\mathrm{R}^{2}\right)$ of 1.13 and 0.96 , respectively (Fig. 1C). In terms of refractive accuracy, 93 and $100 \%$ of eyes showed an achieved SEQ within \pm 0.50 diopters (D) and $\pm 1.0 \mathrm{D}$, respectively (Fig. 1D). The preoperative refractive astigmatism was within $0.50 \mathrm{D}$ and $1.00 \mathrm{D}$ in 70 and $100 \%$ of eyes, respectively, while the postoperative astigmatism was within $0.50 \mathrm{D}$ and $1.00 \mathrm{D}$ in 87 and $97 \%$ of eyes, respectively (Fig. 1E).

\section{Discussion}

This study presented ICL implantation to be an effective, safe, and predictable method to treat myopic regression in eyes underwent previous LVC. 3-month postoperative mean UDVA and CDVA were significantly improved after ICL implantation, and $97 \%$ of eyes showed no change or gain of Snellen lines of CDVA. Also, 93\% of eyes showed an achieved SEQ within $\pm 0.50 \mathrm{D}$. Several mechanisms are suggested to be related to myopic regression after $\mathrm{LVC}$, but the exact mechanism leading to regression is yet to be elucidated. Among these mechanisms, corneal epithelial remodeling and corneal stromal healing response are major factors for myopic regression $[1,11,12]$. Compensatory epithelial hyperplasia and a forward shift of both anterior and posterior corneal surfaces can lead to myopic regression following LVC.

Secondary corneal surgeries to treat myopic regression includes the flap re-lift LASIK and surface ablation [13]. These types of surgeries can induce aggressive

Table 1 Characteristics of eyes which underwent implantable collamer lens implantation after previous laser vision correction surgery

\begin{tabular}{|c|c|c|c|}
\hline Characteristics & Preop & 3-month postop & $P$ \\
\hline Age (years) & $35.00 \pm 4.48(27$ to 43$)$ & & \\
\hline No. of eyes (right/left) & $15 / 15$ & & \\
\hline $\operatorname{Sex}(M / F)$ & $8 / 22$ & & \\
\hline Type of previous surgery (PRK/LASIK) & $21 / 9$ & & \\
\hline Time interval after previous surgery (months) & $73.27 \pm 6.70(61$ to 84$)$ & & \\
\hline Central corneal thickness $(\mu \mathrm{m})$ & $\begin{array}{l}456.03 \pm 35.71 \\
(413 \text { to } 524)\end{array}$ & $\begin{array}{l}456.77 \pm 35.48 \\
(412 \text { to } 520)\end{array}$ & 0.647 \\
\hline Mean keratometry (D) & $\begin{array}{l}38.96 \pm 1.39 \\
\text { (35.63 to } 41.13)\end{array}$ & $\begin{array}{l}38.90 \pm 1.52 \\
\text { (35.13 to } 41.88)\end{array}$ & 0.295 \\
\hline Endothelial cell count $\left(/ \mathrm{mm}^{2}\right)$ & $3006 \pm 290$ (2480 to 3524$)$ & $2999 \pm 367$ (2314 to 3771$)$ & 0.841 \\
\hline LogMAR UDVA & $0.70 \pm 0.33$ (0.10 to 1.30$)$ & $-0.03 \pm 0.11(-0.18$ to 0.30$)$ & $<0.001^{*}$ \\
\hline LogMAR CDVA & $0.01 \pm 0.04$ (0 to 0.15$)$ & $-0.04 \pm 0.09(-0.18$ to 0.22$)$ & $0.001^{*}$ \\
\hline \multicolumn{4}{|l|}{ Refractive errors (D) } \\
\hline Sphere & $-2.32 \pm 1.21(-6.00$ to -0.75$)$ & $0.05 \pm 0.31(-0.50$ to 0.75$)$ & $<0.001^{*}$ \\
\hline Cylinder & $-0.37 \pm 0.39(-1.00$ to 0$)$ & $-0.23 \pm 0.40(-1.25$ to 0$)$ & 0.084 \\
\hline Spherical equivalent & $-2.50 \pm 1.22(-6.25$ to -1.13$)$ & $-0.06 \pm 0.33(-0.63$ to 0.75$)$ & $<0.001^{*}$ \\
\hline
\end{tabular}




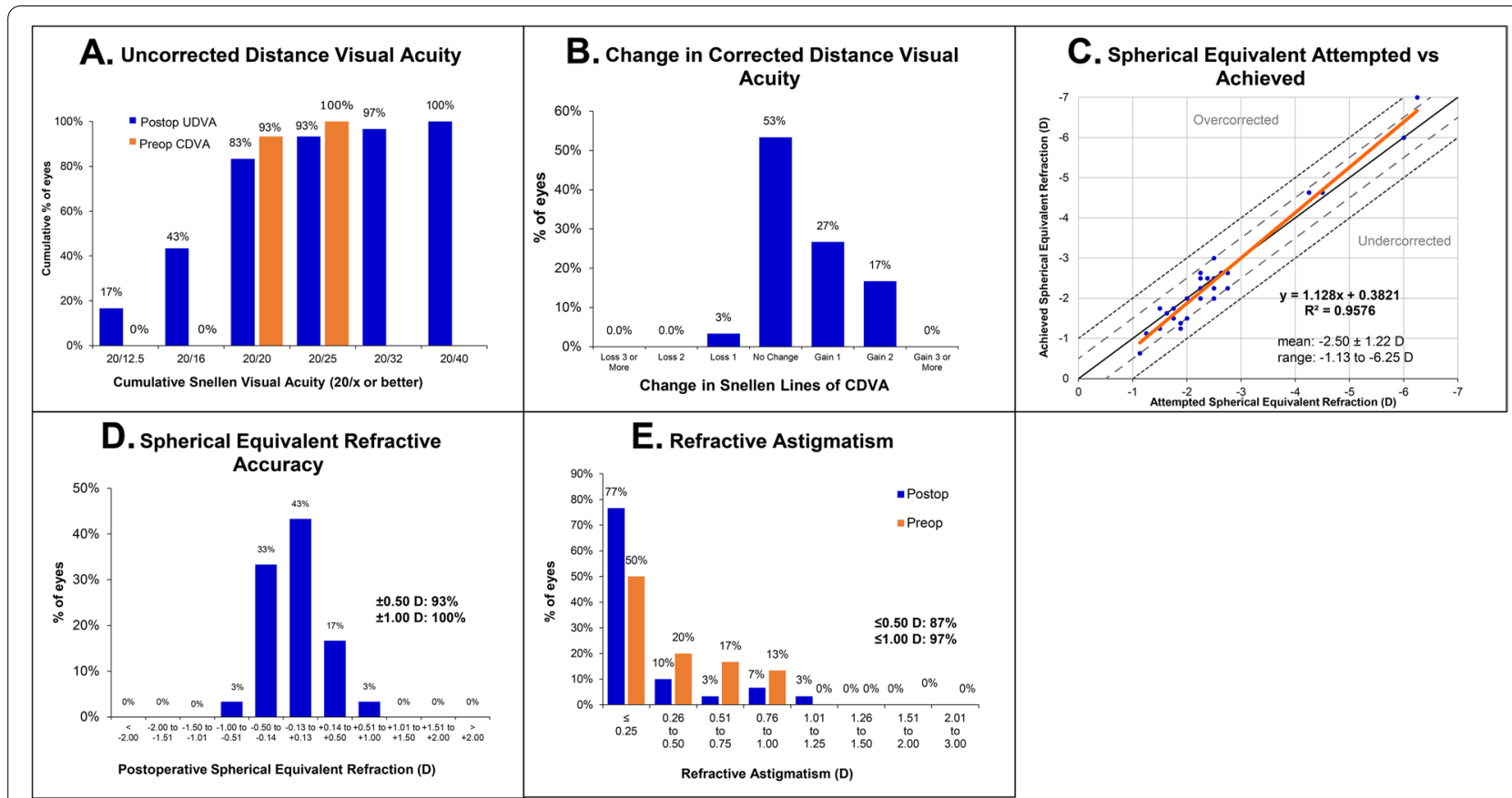

Fig. 1 Visual and refractive outcomes after implantable collamer lens implantation (ICL) in eyes with myopic regression after primary laser vision correction surgery. A Cumulative 3-month postoperative uncorrected distance visual acuity (UDVA) and preoperative corrected distance visual acuity (CDVA). B Changes in Snellen lines of postoperative CDVA relative to the preoperative CDVA. C The attempted versus achieved changes in spherical equivalent refraction (SEQ) 3 months after ICL implantation. D Accuracy of SEQ relative to the intended target. E Preoperative and postoperative refractive astigmatism. $\mathrm{D}=$ diopters

corneal wound healing response and pose a risk of epithelial ingrowth and corneal haze formation. Furthermore, increased risk of corneal ectasia and induction of higher-order aberrations can negatively affect the outcomes of the procedure. Surgical retreatment using phakic intraocular lens implantation can naturally avoid corneal complications. Fast visual recovery and relatively less postoperative pain are also expected. Several studies have reported the outcomes of phakic intraocular lens implantation after previous corneal surgeries, including radial keratotomy, LASIK, and PRK $[8,10,14,15]$. ICL implantation was reportedly effective for both myopic and hyperopic regression. Stable refractive outcomes up to 5 years after ICL implantation was also reported in one of the studies [8]. In our study, ICL implantation in eyes with myopic regression showed safe, effective, and predictable outcomes in larger sample compared to the previous study [8]. The 3-month Snellen UDVA was better than $20 / 20$ for $83 \%$ of eyes, and the safety and efficacy indices at 3 months postoperatively were $1.13 \pm 0.20$ and $1.11 \pm 0.22$, respectively. These results were similar to or better than those of a previous study in which $52.6 \%$ of eyes showed a UDVA better than $20 / 20$ at 1 week-1 month postoperatively [8]. In addition, there was no clinically significant difference in the postoperative outcomes according to the type of primary LVC surgery including PRK and LASIK. As the size of each group was relatively small, generalizing these results is difficult. Further investigation in a large sample will be helpful to investigate results of enhancement by ICL implantation according to types of primary LVC surgery. Calculation of ICL power is based on the vertex formula modified by Feingold and Olsen [16-18]. Mean corneal power, corneal thickness and the anterior chamber depth are needed to calculate the power of the ICL. As the ratio of the anterior and posterior corneal curvature changes after LVC surgery, it could be a source of error in ICL power calculation. Despite previous corneal surface modification, the modified vertex formula showed predictable outcomes; 93 and 100\% of eyes showed an SEQ within \pm 0.5 and $\pm 1.0 \mathrm{D}$, respectively. There was no significant change in the cylindrical error after ICL implantation. This may be owing to the fact that toric ICL was not implanted in any of the cases in our study. A probable source of the refractive astigmatism modification is the surgical induced astigmatism.

Limitations of the present study include a short follow-up period and small sample size. We only included patients with stable refractive errors for at least 1 year to clinically exclude myopic progression, however, 
Table 2 Characteristics of eyes which underwent implantable collamer lens implantation after previous laser vision correction surgery according to the types of previous laser vision correction

\begin{tabular}{|c|c|c|c|}
\hline Type of previous LVC & PRK $(n=21)$ & LASIK $(n=9)$ & $P$ \\
\hline No. of eyes (right/left) & $11 / 10$ & $4 / 5$ & $>0.999$ \\
\hline Age (years) & $33.38 \pm 4.04$ & $38.78 \pm 3.00$ & $0.001^{*}$ \\
\hline $\operatorname{Sex}(M / F)$ & $6 / 15$ & $2 / 7$ & $>0.999$ \\
\hline Time interval after previous surgery (months) & $71.29 \pm 5.82$ & $77.89 \pm 6.59$ & $0.022^{*}$ \\
\hline Central corneal thickness $(\mu \mathrm{m})$ & $452.95 \pm 34.69$ & $463.22 \pm 39.12$ & 0.455 \\
\hline Preoperative mean keratometry (D) & $38.97 \pm 1.50$ & $38.94 \pm 1.18$ & 0.700 \\
\hline \multicolumn{4}{|l|}{ LogMAR UDVA } \\
\hline Preoperative & $0.70 \pm 0.28$ & $0.69 \pm 0.43$ & 0.982 \\
\hline 3-month postoperative & $-0.04 \pm 0.07$ & $0 \pm 0.16$ & 0.657 \\
\hline P (preop vs 3-month postop) & $<0.001$ & 0.008 & \\
\hline \multicolumn{4}{|l|}{ LogMAR CDVA } \\
\hline Preoperative & 0 & $0.03 \pm 0.07$ & 0.349 \\
\hline 3-month postoperative & $-0.05 \pm 0.06$ & $0 \pm 0.14$ & 0.563 \\
\hline$P$ (preop vs 3-month postop) & 0.006 & 0.114 & \\
\hline \multicolumn{4}{|l|}{ Spherical error (D) } \\
\hline Preoperative & $-2.00 \pm 0.48$ & $-3.08 \pm 1.95$ & 0.504 \\
\hline 3-month postoperative & $-0.02 \pm 0.28$ & $0.22 \pm 0.32$ & 0.051 \\
\hline P (preop vs 3-month postop) & $<0.001$ & 0.008 & \\
\hline \multicolumn{4}{|l|}{ Cylindrical error (D) } \\
\hline Preoperative & $-0.37 \pm 0.42$ & $-0.36 \pm 0.33$ & 0.981 \\
\hline 3-month postoperative & $-0.24 \pm 0.39$ & $-0.19 \pm 0.27$ & 0.918 \\
\hline P (preop vs 3-month postop) & 0.146 & 0.301 & \\
\hline \multicolumn{4}{|l|}{ Spherical equivalent (D) } \\
\hline Preoperative & $-2.17 \pm 0.42$ & $-3.26 \pm 2.00$ & 0.733 \\
\hline 3-month postoperative & $-0.14 \pm 0.31$ & $0.13 \pm 0.30$ & 0.050 \\
\hline P (preop vs 3-month postop) & $<0.001$ & 0.008 & \\
\hline \multicolumn{4}{|l|}{ Endothelial cell count (/mm²) } \\
\hline Preoperative & $2986 \pm 314$ & $3053 \pm 233$ & 0.556 \\
\hline 3-month postoperative & $2966 \pm 388$ & $3076 \pm 321$ & 0.319 \\
\hline P (preop vs 3-month postop) & 0.476 & 0.859 & \\
\hline
\end{tabular}

PRK photorefractive keratectomy; LASIK laser in situ keratomileusis; $D$ diopters; UDVA uncorrected distance visual acuity; CDVA corrected distance visual acuity;Values are presented as mean \pm standard deviation (range)* significant difference between previous PRK and LASIK group

there is possibility that myopic progression cases were included as our study did not include evaluation of keratometry and axial length changes before and after the primary LVC surgery. Other assessment modalities, including assessment of wavefront aberrations, will be helpful to analyze the outcomes of ICL implantation after LVC surgeries.

\section{Conclusions}

ICL implantation showed safe, effective, and predictable postoperative outcomes in eyes with myopic regression. In addition, there was no significantly different result based on the type of previous LVC surgery, including PRK and LASIK. ICL implantation can be considered as an appropriate option to treat myopic regression after LVC surgery.

\section{Acknowledgments}

None.

\section{Authors' contributions}

BC and JHK acquired, analyzed and interpreted the patient data, and drafted the manuscript. These 2 authors contributed equally to this study. DSYK contributed to the conception of this study, interpreted the patient data.

DJK acquired and analyzed the data. KYS, EKK contributed to the conception of this study, interpreted the patient data. IJ contributed to the conception of this study, interpreted the patient data. TK contributed to the conception of this study, interpreted the patient data, and revised the manuscript. The author(s) read and approved the final manuscript.

\section{Funding}

This research was Supported by the Basic Science Research Program (NRF2021R111A1A01047951) of the National Research Foundation (NRF) funded 
by the Ministry of Science, ICT, and Future Planning. The funding organization had no role in the design or conduct of this study.

\section{Availability of data and materials}

Not applicable.

\section{Declarations}

\section{Ethics approval and consent to participate}

The tenets of the Declaration of Helsinki and good clinical practice were followed, and institutional review board approval from Yonsei University College of Medicine was obtained (No. 4-2020-0396). Owing to the retrospective nature of the study, the requirement for informed consent was waived.

\section{Consent for publication}

Not applicable.

\section{Competing interests}

Dr. Kang is a consultant for Avedro Inc., Schwind eye-tech-solutions, and Carl Zeiss Meditec AG. The remaining authors have no proprietary or financial interest in the materials presented herein.

\section{Author details}

'Eyereum Eye Clinic, Seoul, Republic of Korea. ${ }^{2}$ Apgujeong Eye Clinic, Seoul, Republic of Korea. ${ }^{3}$ Department of Ophthalmology, Institute of Vision Research, Yonsei University College of Medicine, 50-1, Yonsei-ro, Seodaemun-gu, Seoul 03722, Republic of Korea.

Received: 24 April 2021 Accepted: 30 October 2021

Published online: 16 November 2021

\section{References}

1. Chayet AS, Assil KK, Montes M, Espinosa-Lagana M, Castellanos A, Tsioulias G. Regression and its mechanisms after laser in situ keratomileusis in moderate and high myopia. Ophthalmology. 1998;105(7):1194-9.

2. Saeed A, O'Doherty M, O'Doherty J, O'Keefe M. Analysis of the visual and refractive outcome following laser in situ keratomileusis (LASIK) retreatment over a four-year follow-up period. Int Ophthalmol. 2007;27(1):23-9.

3. Brahma A, McGhee CN, Craig JP, Brown AD, Weed KH, McGhee J, et al. Safety and predictability of laser in situ keratomileusis enhancement by flap reelevation in high myopia. J Cataract Refract Surg. 2001;27(4):593-603.

4. Pokroy R, Mimouni M, Sela T, Munzer G, Kaiserman I. Myopic laser in situ keratomileusis retreatment: incidence and associations. J Cataract Refract Surg. 2016;42(10):1408-14.
5. Mohammadi SF, Nabovati P, Mirzajani A, Ashrafi E, Vakilian B. Risk factors of regression and undercorrection in photorefractive keratectomy: a case-control study. Int J Ophthalmol. 2015;8(5):933-7.

6. Pokroy R, Mimouni M, Sela T, Munzer G, Kaiserman I. Predictors of myopic photorefractive keratectomy retreatment. J Cataract Refract Surg. 2017;43(6):825-32.

7. Nakamura T, Isogai N, Kojima T, Yoshida Y, Sugiyama Y. Posterior chamber Phakic intraocular Lens implantation for the correction of myopia and myopic astigmatism: a retrospective 10-year follow-up study. Am J Ophthalmol. 2019;206:1-10.

8. Chen X, Wang XY, Zhang X, Chen Z, Zhou XT. Implantable collamer lens for residual refractive error after corneal refractive surgery. Int J Ophthalmol. 2016;9(10):1421-6.

9. Alfonso JF, Lisa C, Alfonso-Bartolozzi B, Fernandez-Vega-Cueto L, MontesMico R. Implantable Collamer Lens((R)) for Management of Pseudophakic Ametropia in eyes with a Spectrum of previous corneal surgery. J Refract Surg. 2018;34(10):654-63.

10. Kamiya K, Shimizu K. Implantable Collamer lens for hyperopia after radial keratotomy. J Cataract Refract Surg. 2008;34(8):1403-4.

11. Chan TC, Liu D, Yu M, Jhanji V. Longitudinal evaluation of posterior corneal elevation after laser refractive surgery using swept-source optical coherence tomography. Ophthalmology. 2015;122(4):687-92.

12. Jaycock PD, Lobo L, Ibrahim J, Tyrer J, Marshall J. Interferometric technique to measure biomechanical changes in the cornea induced by refractive surgery. J Cataract Refract Surg. 2005;31 (1):175-84.

13. Schallhorn SC, Venter JA, Hannan SJ, Hettinger KA, Teenan D. Flap lift and photorefractive keratectomy enhancements after primary laser in situ keratomileusis using a wavefront-guided ablation profile: refractive and visual outcomes. J Cataract Refract Surg. 2015;41(11):2501-12.

14. Kamiya K, Shimizu K, Komatsu M. Implantable Collamer Lens implantation and limbal relaxing incisions for the correction of hyperopic astigmatism after laser in situ keratomileusis. Cornea. 2010;29(1):99-101.

15. Srinivasan S, Drake A, Herzig S. Early experience with implantable collamer lens in the management of hyperopia after radial keratotomy. Cornea. 2008;27(3):302-4.

16. Fyodorov SN, Galin MA, Linksz A. Calculation of the optical power of intraocular lenses. Investig Ophthalmol. 1975;14(8):625-8.

17. Olsen T, Corydon L, Gimbel H. Intraocular lens power calculation with an improved anterior chamber depth prediction algorithm. J Cataract Refract Surg. 1995;21(3):313-9.

18. Olsen T, Thim K, Corydon L. Accuracy of the newer generation intraocular lens power calculation formulas in long and short eyes. J Cataract Refract Surg. 1991;17(2):187-93.

\section{Publisher's Note}

Springer Nature remains neutral with regard to jurisdictional claims in published maps and institutional affiliations.
Ready to submit your research? Choose BMC and benefit from:

- fast, convenient online submission

- thorough peer review by experienced researchers in your field

- rapid publication on acceptance

- support for research data, including large and complex data types

- gold Open Access which fosters wider collaboration and increased citations

- maximum visibility for your research: over 100M website views per year

At BMC, research is always in progress.

Learn more biomedcentral.com/submissions 